\title{
BMJ Open Association of erythrocyte parameters with metabolic syndrome in the Pearl River Delta region of China: a cross sectional study
}

\author{
Ling Ling Huang, ${ }^{1}$ Dong-Mei Dou, ${ }^{1}$ Nan Liu, ${ }^{2}$ Xiao Xiao Wang, ${ }^{1}$ Li-Ying Fu, ${ }^{1}$ \\ Xiao Wu, ${ }^{1}$ Peixi Wang ${ }^{1,2}$
}

To cite: Huang LL, Dou D-M, Liu N, et al. Association of erythrocyte parameters with metabolic syndrome in the Pearl River Delta region of China: a cross sectional study. BMJ Open 2018;8:e019792. doi:10.1136/ bmjopen-2017-019792

- Prepublication history for this paper is available online. To view these files, please visit the journal online (http://dx.doi. org/10.1136/bmjopen-2017019792).

Huang LL and D-MD contributed equally.

Received 26 September 2017 Revised 14 November 2017 Accepted 29 November 2017

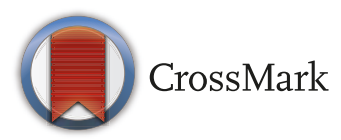

${ }^{1}$ Institute of Chronic Disease Risks Assessment, Henan University, Kaifeng, China ${ }^{2}$ School of Public Health, Guangzhou Medical University, Guangzhou, China

Correspondence to Dr Peixi Wang; peixi001@163.com

\section{ABSTRACT}

Objective Increasing studies have reported that erythrocyte parameters, including red blood cells (RBCs), haematocrit $(\mathrm{HCT})$, haemoglobin $(\mathrm{Hb})$ and red blood cell distribution width (RDW), are associated with metabolic syndrome (MetS) in adults worldwide. However, the association, stratified by sex, remains to be elucidated, particularly in the Pearl River Delta region of China. Therefore, our aim was to explore the association of erythrocyte parameters with MetS, stratified by sex, in the Pearl River Delta region of China.

Methods In this cross sectional study, 2161 men and 2511 women were enrolled. MetS was diagnosed using a modified version of the Adult Treatment Panel III criteria. Logistic regression analyses were performed to calculate adjusted ORs of erythrocyte parameters associated with MetS stratified by sex.

Results The prevalence of MetS was higher in women than in men (35.2\%vs26.7\%). RBC, HCT, Hb and RDW values increased linearly with the number of MetS components from 0 to 5 identified in both men and women. Among men, the ORs of MetS risk increased across the tertiles of $\mathrm{Hb}(\mathrm{Q} 2: \mathrm{OR}=1.921,95 \% \mathrm{Cl}=1.170$ to 3.151; Q3: $\mathrm{OR}=1.992,95 \% \mathrm{Cl}=1.198$ to 3.312 ). Men in the highest tertiles of RDW had a 2.752-fold increased risk of suffering from MetS compared with those in the reference group. Among women, the ORs of MetS risk also increased across the tertiles of $\mathrm{Hb}(\mathrm{Q} 2: \mathrm{OR}=1.538,95 \% \mathrm{Cl}=1.008$ to 2.348; Q3: $\mathrm{OR}=1.665,95 \% \mathrm{Cl}=1.075$ to 2.578$)$. Women in the highest tertiles of RBC had a 1.718-fold increased risk of experiencing MetS compared with those in the reference group.

Conclusions MetS was more prevalent in women than in men. The association between erythrocyte parameters and MetS differed between the sexes. RBC and $\mathrm{Hb}$ were identified as risk factors for MetS in women and $\mathrm{Hb}$ and RDW as risk factors in men.

\section{INTRODUCTION}

Metabolic syndrome (MetS) is defined as a cluster of multiple correlated metabolic features, including abdominal obesity, hypertension, elevated triglyceride (TG) levels, decreased high density lipoprotein

\section{Strengths and limitations of this study}

- A large sample of subjects were enrolled in our survey.

- To the best of our knowledge, this is the first study to report the association of erythrocyte parameters with MetS, stratified by sex, in the Pearl River Delta region of China.

- The present study was designed as a cross sectional study; therefore, direct causation may not be concluded from the results.

- Supplementary information about the lifestyle of the subjects was not collected; therefore, these factors could not be included in the adjustments of our multivariate logistic regression analyses.

cholesterol (HDL-C) levels and hyperglycaemia. ${ }^{1}$ It is known to be strongly associated with an increased risk of type 2 diabetes, ${ }^{2}$ cardiovascular disease $\mathrm{e}^{2-4}$ and all cause mortality. ${ }^{4}$ In recent years, MetS has emerged as a global public health issue owing to its increased prevalence around the world, affecting nearly $20-30 \%$ of adults in many countries. $^{5-7}$ Hence early identification of individuals at high risk of MetS is essential for the prevention of MetS.

Currently, the pathogenesis of MetS is not clearly understood. Generally, MetS is accompanied by insulin resistance and/or chronic low grade inflammation. ${ }^{89}$ Numerous investigators previously reported that erythrocyte parameters, including red blood cell (RBC) count, haematocrit (HCT), haemoglobin $(\mathrm{Hb})$ and red blood cell distribution width (RDW) were positively associated with insulin resistance and chronic low grade inflammation. ${ }^{10-14}$ In fact, RBC, ${ }^{14-16}$ HCT, ${ }^{15} 16$ $\mathrm{Hb}^{141517}$ and $\mathrm{RDW}^{18}$ were demonstrated in several studies worldwide to correlate with MetS in adults. However, the association between erythrocyte parameters and MetS 
remains controversial, because the results reported are inconsistent depending on the different ethnic populations studied. In addition, discrepancies in the results may be partly attributed to differences between the sexes. Many studies simply applied sex as an adjustment variable to investigate the relationship between erythrocyte parameters and MetS, and no studies were conducted in the Pearl River Delta region of China. Therefore, the aim of this study was to explore the association between erythrocyte parameters and MetS, stratified by sex, in the Pearl River Delta region of China.

\section{MATERIALS AND METHODS Study participants}

This cross sectional study involved participants who underwent a general health examination at the Community Health Service Agencies in the Pearl River Delta region of China in 2015. The health examination included recording of medical history, anthropometric measurements and laboratory tests. Participants with a history of cardiovascular diseases, severe liver or kidney dysfunction, tumours or severe inflammatory diseases were excluded. In addition, participants who did not have complete data on their MetS components and erythrocyte parameters were excluded. A total of 4672 subjects (2161 men and 2511 women) were enrolled in the study. The study was approved by the ethics committee of Guangdong Sociological Society. Written informed consent was obtained from all participants.

\section{Data collection and measurements}

Medical histories of subjects were obtained by review of self-reported questionnaires. Anthropometric parameters were measured by trained staff, following a standardised protocol. Height, weight, waist circumference (WC), systolic blood pressure (SBP) and diastolic blood pressure (DBP) were measured several times, and all mean values of the above indexes were calculated. Body mass index (BMI) was calculated as weight $(\mathrm{kg})$ divided by height $\left(\mathrm{m}^{2}\right)$. After an overnight fast, venous blood samples from participants were obtained and analysed for TG, total cholesterol (TC), HDL-C, low density lipoprotein cholesterol (LDL-C), fasting plasma glucose (FPG), uric acid (UA), white blood cell (WBC) count, platelets (PLT), RBC count, HCT, Hb, RDW, alanine transaminase (ALT), aspartate aminotransferase (AST), $\gamma$-glutamyl transferase (GGT), albumin (ALB) and glycated haemoglobin A1c (HbAIc).

\section{Quality control}

All data were collected by trained doctors or nurses who checked the data from every participant. In addition, several supervisors verified the authenticity of the data.

\section{Tertiles of erythrocyte parameters levels}

Erythrocyte parameter levels were categorised into tertiles on the basis of individual distributions, for men and women (in men: RBC, Q1 $<4.37 \times 10^{12} / \mathrm{L}, \mathrm{Q} 2=4.37 \sim 4,75 \times 10^{12} / \mathrm{L}$, Q3 $\geq 4.76 \times 10^{12} / \mathrm{L}$; HCT, Q1 $<39.8 \%$, Q2=39.8 42.4\%, Q3 $\geq 42.5 \%$; Hb, Q1 $<137 \mathrm{~g} / \mathrm{L}, \quad \mathrm{Q} 2=137 \sim 146 \mathrm{~g} / \mathrm{L}$, Q3 $\geq 147 \mathrm{~g} / \mathrm{L}$; RDW, Q1 $<12.5 \%$, Q2 $=12.5 \sim 13.1 \%$, Q3 $\geq 13.2 \%$; in women: $\mathrm{RBC}, \mathrm{Q} 1<3.96 \times 10^{12} / \mathrm{L}, \mathrm{Q} 2=3.96 \sim 4.27 \times 10^{12} / \mathrm{L}$, $\mathrm{Q} 3 \geq 4.28 \times 10^{12} / \mathrm{L} ; \mathrm{HCT}, \mathrm{Q} 1<35.2 \%, \mathrm{Q} 2=35.2 \sim 37.3 \%$, Q3 $\geq 37.4 \%$; Hb, Q1 <120 g/L, Q2=120 127 g/L, Q3 $\geq 128 \mathrm{~g} / \mathrm{L}$; RDW, Q1 $<12.3 \%$, Q2=12.3 12.8\%, Q3 $\geq 12.9 \%$ ).

\section{Definition of metabolic syndrome}

MetS was diagnosed using a modified version of the Adult Treatment Panel III (ATP III) criteria, ${ }^{1}$ which included at least three of the following five components: (1) $\mathrm{WC} \geq 90 \mathrm{~cm}$ in men and $\mathrm{WC} \geq 80 \mathrm{~cm}$ in women; (2) SBP $\geq 130 \mathrm{~mm} \mathrm{Hg}$ or DBP $\geq 85 \mathrm{~mm} \mathrm{Hg}$; (3) TG $\geq 1.70 \mathrm{mmol} / \mathrm{L}$; (4) HDL-C $<1.03 \mathrm{mmol} / \mathrm{L}$ in men and HDL-C $<1.29 \mathrm{mmol} / \mathrm{L}$ in women; and (5) $\mathrm{FPG} \geq 5.6 \mathrm{mmol} / \mathrm{L}$.

\section{Statistical analysis}

All statistical analyses were performed using the Statistical Package for the Social Sciences (SPSS) V.21.0 (SPSS Inc, Chicago, Illinois, USA). Data are presented as mean $\pm \mathrm{SD}$ or frequency (percentage). The t test was used to evaluate differences in characteristics of the study subjects with and without MetS stratified by sex. The $\chi^{2}$ test was performed to compare the proportion of MetS components, from 0 to 5 , between men and women, and to compare the prevalence of MetS dependent on the tertiles for RBC count, HCT, Hb and RDW between men and women. A one way ANOVA was conducted to test mean levels for erythrocyte parameters according to the number of MetS components in men and women separately. Multivariate logistic regression analyses (the enter selection procedure) were performed to calculate adjusted ORs for the erythrocyte parameters associated with MetS, stratified by sex, with adjustments for potential confounders (the statistically significant variables in table 1-men: adjusted for age, WC, SBP, DBP, TG, HDL-C, FPG, ALT, AST, GGT, BMI, UA, WBC, PLT and HbAIc; women: adjusted for age, WC, SBP, DBP, TG, HDL-C, FPG, ALT, GGT, BMI, TC, LDL-C, UA, WBC, PLT and $\mathrm{HbAIc}$ ). A $\mathrm{P}$ value $<0.05$ was considered to be statistically significant.

\section{RESULTS \\ Prevalence of MetS}

In total, there were 2161 men and 2511 women enrolled in the study, of whom 576 men $(26.7 \%)$ and 885 women $(35.2 \%)$ were diagnosed with MetS.

\section{Characteristics of the study subjects}

In this study, among men, the mean age of the MetS group was significantly lower than that of the non-MetS group, whereas the opposite trend was observed among women $(\mathrm{P}<0.001)$. In the cluster of MetS components, WC, SBP, DBP, TG and FPG levels were much greater in the MetS group than in the non-MetS group in both 
Table 1 Characteristics of the study subjects with and without metabolic syndrome, stratified by sex

\begin{tabular}{|c|c|c|c|c|c|c|}
\hline \multirow[b]{2}{*}{ Variable } & \multicolumn{3}{|l|}{ Men $(n=2161)$} & \multicolumn{3}{|c|}{ Women $(n=2511)$} \\
\hline & MetS & Non-MetS & $P$ value & MetS & Non-MetS & $P$ value \\
\hline MetS status (n (\%)) & $576(26.7)$ & 1585 (73.3) & & $885(35.2)$ & $885(35.2)$ & \\
\hline \multicolumn{7}{|c|}{ Components of MetS } \\
\hline WC (cm) & $89.98 \pm 6.79$ & $82.40 \pm 7.76$ & $<0.001$ & $85.94 \pm 7.21$ & $77.59 \pm 8.56$ & $<0.001$ \\
\hline $\mathrm{DBP}(\mathrm{mm} \mathrm{Hg})$ & $89.24 \pm 10.47$ & $82.93 \pm 11.09$ & $<0.001$ & $84.13 \pm 10.25$ & $78.51 \pm 10.53$ & $<0.001$ \\
\hline $\mathrm{TG}(\mathrm{mmol} / \mathrm{L})$ & $2.76 \pm 1.77$ & $1.29 \pm 0.91$ & $<0.001$ & $2.15 \pm 1.41$ & $1.20 \pm 1.87$ & $<0.001$ \\
\hline HDL-C (mmol/L) & $1.00 \pm 0.47$ & $1.28 \pm 0.44$ & $<0.001$ & $1.17 \pm 0.22$ & $1.50 \pm 0.34$ & $<0.001$ \\
\hline $\mathrm{FPG}(\mathrm{mmol} / \mathrm{L})$ & $5.53 \pm 2.01$ & $4.87 \pm 1.40$ & $<0.001$ & $5.38 \pm 1.86$ & $4.71 \pm 0.97$ & $<0.001$ \\
\hline \multicolumn{7}{|c|}{ Erythrocyte parameters } \\
\hline $\mathrm{Hb}(\mathrm{g} / \mathrm{L})$ & $147.11 \pm 12.57$ & $139.02 \pm 12.68$ & $<0.001$ & $129.68 \pm 14.45$ & $121.50 \pm 11.82$ & $<0.001$ \\
\hline RDW (\%) & $13.33 \pm 0.96$ & $12.87 \pm 1.21$ & $<0.001$ & $13.18 \pm 1.90$ & $12.88 \pm 2.27$ & $<0.001$ \\
\hline \multicolumn{7}{|c|}{ Liver function parameters } \\
\hline $\operatorname{ALT}(\mathrm{u} / \mathrm{L})$ & $31.44 \pm 18.35$ & $26.31 \pm 15.52$ & $<0.001$ & $24.09 \pm 13.81$ & $21.14 \pm 11.79$ & $<0.001$ \\
\hline AST (u/L) & $26.37 \pm 15.87$ & $24.80 \pm 10.00$ & 0.026 & $23.82 \pm 8.90$ & $23.27 \pm 8.63$ & 0.129 \\
\hline GGT (u/L) & $48.73 \pm 39.88$ & $36.04 \pm 26.83$ & $<0.001$ & $32.00 \pm 22.79$ & $26.12 \pm 26.03$ & $<0.001$ \\
\hline ALB (g/L) & $47.36 \pm 3.23$ & $47.32 \pm 4.07$ & 0.820 & $47.48 \pm 4.32$ & $47.77 \pm 12.28$ & 0.484 \\
\hline \multicolumn{7}{|c|}{ Other clinical characteristics } \\
\hline BMI $\left(\mathrm{kg} / \mathrm{m}^{2}\right)$ & $25.90 \pm 2.67$ & $23.61 \pm 3.04$ & $<0.001$ & $25.21 \pm 3.05$ & $22.85 \pm 3.17$ & $<0.001$ \\
\hline
\end{tabular}

Data are presented as mean \pm SD or $n(\%)$.

ALB, albumin; ALT, alanine transaminase; AST, aspartate aminotransferase; BMI, body mass index; DBP, diastolic blood pressure ; FPG, fasting plasma glucose; GGT, $\gamma$-glutamyl transferase; Hb, haemoglobin; HbAlc, glycated haemoglobin A1c; HCT, haematocrit; HDL-C, high density lipoprotein cholesterol; LDL-C, low density lipoprotein cholesterol; MetS, metabolic syndrome; PLT, platelet; RBC, red blood cell; RDW, red blood cell distribution width; SBP, systolic blood pressure; TC, total cholesterol; TG, triglyceride; UA, uric acid; WBC, white blood cell; WC, waist circumference.

men and women, but HDL-C levels were significantly lower in the MetS group than that those in the non-MetS group in both men and women $(\mathrm{P}<0.001)$. In the cluster of erythrocyte parameters, we found that RBC, HCT, $\mathrm{Hb}$ and RDW were significantly higher in the MetS group than in the non-MetS group in both men and women $(\mathrm{P}<0.001)$. Additional information on the characteristics of study subjects with and without MetS, stratified by sex, are presented in table 1 .

\section{Proportion of MetS components}

Our results revealed that most men experienced one metabolic disorder, and most women suffered from two metabolic disorders. In addition, the proportion of MetS components from 2 to 5 was significantly lower in men than that in women $(25.5 \%$ vs $27.8 \%, 18 \%$ vs $21.3 \%$, $7.5 \%$ vs $11.3 \%$ and $1.1 \%$ vs 2.6 , respectively). Additional information is shown in figure 1.

\section{Association of erythrocyte parameters with MetS}

The study showed that the levels of RBC, HCT, Hb and RDW clearly increased with the number of MetS components from 0 to 5 identified in both men and women $(P<0.001$, shown in table 2$)$. Figure 2 shows that the prevalence of MetS increased in a dose dependent manner as the tertiles for RBC, HCT, Hb and RDW levels increased in both men and women. Furthermore, at each tertile for the above mentioned parameters, the prevalence of MetS was lower in men than in women, except at the highest tertiles for RDW levels (figure 2). 


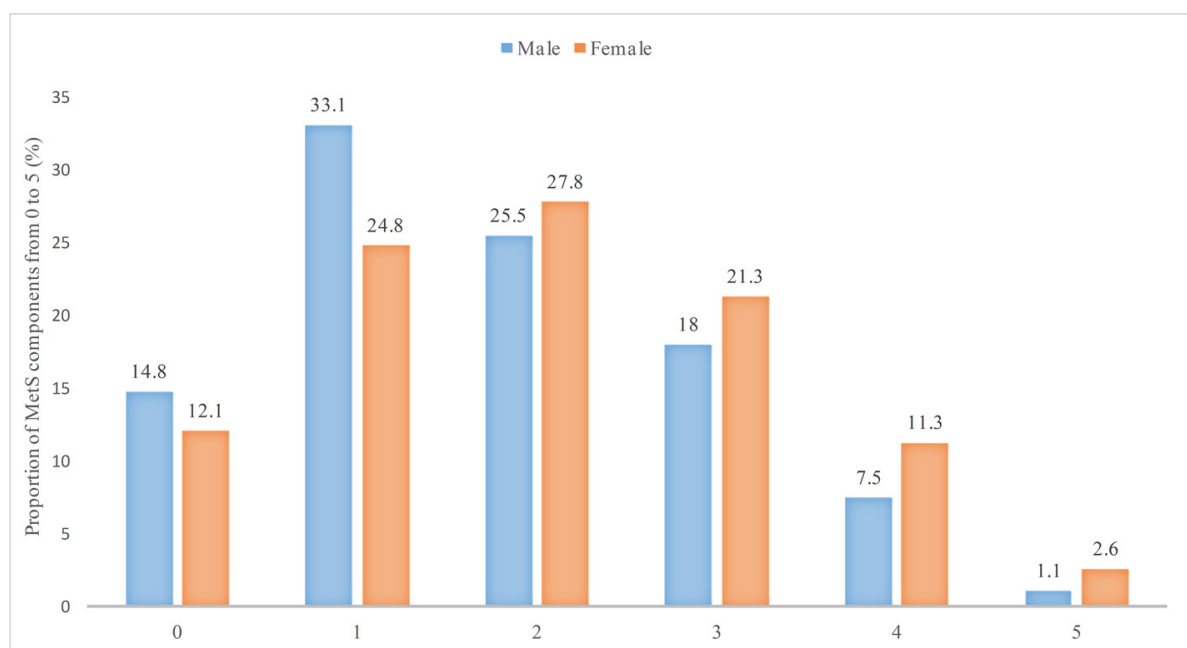

Figure 1 Proportion of metabolic syndrome (MetS) components from 0 to 5 between men and women.

\section{Multivariate logistic regression analysis model}

Adjusted ORs of MetS risk associated with each tertile of RBC, HCT, Hb and RDW are listed in table 3. After adjusting for potential confounders (the statistically significant variables in table $1-$ men: adjusted for age, WC, SBP, DBP, TG, HDL-C, FPG, ALT, AST, GGT, BMI, UA, WBC, PLT and HbAIc; women: adjusted for age, WC, SBP, DBP, TG, HDL-C, FPG, ALT, GGT, BMI, TC, LDL-C, UA, WBC, PLT and HbAIc). A significant association of $\mathrm{Hb}$ and RDW with MetS was observed in men, but this was not the same for RBC and HCT. The ORs of MetS risk increased across the tertiles of $\mathrm{Hb}(\mathrm{Q} 2: \mathrm{OR}=1.921,95 \%$ $\mathrm{CI}=1.170$ to 3.151; $\mathrm{Q} 3: \mathrm{OR}=1.992,95 \% \mathrm{CI}=1.198 \sim 3.312$ ). Men in the highest tertiles of RDW had a 2.752-fold increased risk of suffering from MetS compared with those in the reference group. Only RBC and $\mathrm{Hb}$ levels were observed to be associated with MetS in women. The ORs of MetS risk also increased across the tertiles of $\mathrm{Hb}$ (Q2: $\mathrm{OR}=1.538,95 \% \mathrm{CI}=1.008$ to $2.348 ; \mathrm{Q} 3: \mathrm{OR}=1.665$, $95 \% \mathrm{CI}=1.075$ to 2.578$)$. Women in the highest tertiles of
RBC had a 1.718-fold increased risk of experiencing MetS in comparison with those in the reference group.

\section{DISCUSSION \\ Main findings}

The prevalence of MetS was higher in women than in men $(35.2 \%$ vs $26.7 \%)$. Levels of RBC, HCT, Hb and RDW increased linearly with the number of MetS components from 0 to 5 , identified in both men and women. The association between erythrocyte parameters and MetS differed between the sexes: $\mathrm{Hb}$ and RDW were identified as risk factors for MetS in men and $\mathrm{RBC}$ and $\mathrm{Hb}$ as risk factors in women.

\section{Comparisons with previous studies}

Sex has been demonstrated to be a predictive factor for MetS development. Several studies have shown that women have a higher prevalence of MetS than men. ${ }^{19} 20$ A large scale study conducted in Russia reported that the

Table 2 Levels of erythrocyte parameters in the study subjects according to the number of metabolic syndrome components (from 0 to 5 ) in men and women

\begin{tabular}{|c|c|c|c|c|c|c|c|c|}
\hline Variable & 0 & 1 & 2 & 3 & 4 & 5 & $\mathbf{F}$ & P value \\
\hline \multicolumn{9}{|l|}{ Men } \\
\hline $\mathrm{RBC}$ & $4.44 \pm 0.53$ & $4.55 \pm 0.50$ & $4.55 \pm 0.52$ & $4.80 \pm 0.54$ & $5.31 \pm 0.86$ & $5.95 \pm 1.23$ & 87.448 & $<0.001$ \\
\hline $\mathrm{Hb}$ & $131.60 \pm 12.35$ & $138.81 \pm 12.86$ & $140.71 \pm 12.41$ & $144.51 \pm 11.65$ & $151.28 \pm 12.87$ & $160.04 \pm 9.19$ & 52.445 & $<0.001$ \\
\hline RDW & $12.75 \pm 0.82$ & $12.83 \pm 1.55$ & $13.00 \pm 0.80$ & $13.24 \pm 0.86$ & $13.41 \pm 1.07$ & $14.33 \pm 1.13$ & 20.264 & $<0.001$ \\
\hline $\mathrm{RBC}$ & $4.03 \pm 0.39$ & $4.07 \pm 0.52$ & $4.16 \pm 0.54$ & $4.45 \pm 0.82$ & $4.67 \pm 0.88$ & $4.83 \pm 0.78$ & 66.453 & $<0.001$ \\
\hline $\mathrm{HCT}$ & $35.16 \pm 2.65$ & $35.43 \pm 2.78$ & $35.91 \pm 2.91$ & $37.07 \pm 2.81$ & $37.74 \pm 2.79$ & $37.96 \pm 2.36$ & 52.237 & $<0.001$ \\
\hline $\mathrm{Hb}$ & $119.70 \pm 11.54$ & $121.28 \pm 11.79$ & $122.49 \pm 11.88$ & $128.26 \pm 14.04$ & $130.04 \pm 14.35$ & $139.61 \pm 14.46$ & 59.262 & $<0.001$ \\
\hline RDW & $12.71 \pm 2.10$ & $12.74 \pm 1.40$ & $13.07 \pm 2.87$ & $13.11 \pm 1.39$ & $13.25 \pm 2.70$ & $13.38 \pm 1.13$ & 4.493 & $<0.001$ \\
\hline
\end{tabular}

$\mathrm{Hb}$, haemoglobin; HCT, haematocrit; RBC, red blood cell; RDW, red blood cell distribution width. 
= Male $=$ Female

60

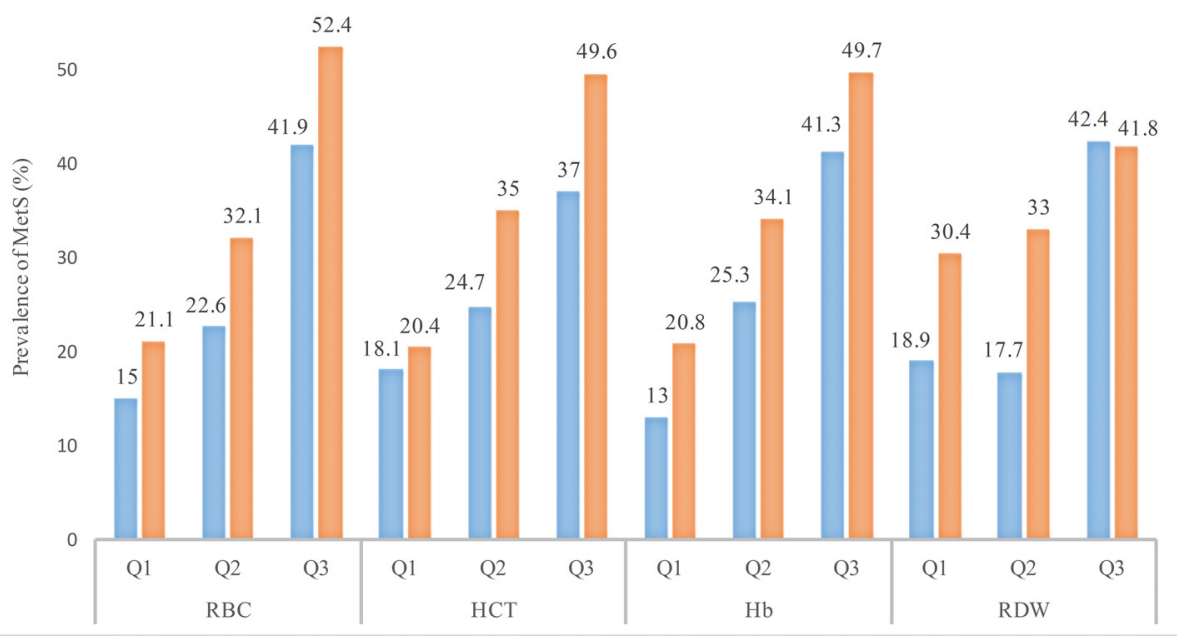

Figure 2 Prevalence of metabolic syndrome (MetS) in association with the tertiles for red blood cell (RBC) count, haematocrit $(\mathrm{HCT})$, haemoglobin $(\mathrm{Hb})$ and red blood cell distribution width $(\mathrm{RDW})$, in men and women separately.

prevalence of MetS diagnosed using the ATP III criteria was $9.5 \%$ in men and $23.5 \%$ in women. ${ }^{19}$ Another study performed in the seven geographical regions of Turkey showed that the prevalence of MetS, as determined by the ATP III criteria, was $28 \%$ in men and $39.6 \%$ in women. ${ }^{20}$ Our study outcomes are in accordance with these reports. However, other studies have reported that men have a higher prevalence of MetS than women. For example, Tao et al found that the 5 year cumulative incidence of MetS in Beijing adults was $14.22 \%$ for men and $7.59 \%$ for women. ${ }^{21}$ Yang et al revealed that the 5 year cumulative incidence of MetS in Taiwanese adults was $14.95 \%$ for men and $9.89 \%$ for women. ${ }^{22}$ Differences in the findings might be due to different study designs and/or the selected populations.

It is well known that MetS represents a cluster of simultaneously occurring metabolic abnormalities. In fact, previous studies demonstrated that $\mathrm{RBC}$ and $\mathrm{Hb}$ levels clearly increased with the number of MetS components, ${ }^{1623}$ and this is demonstrated in our outcomes. It has also been shown that a higher number of MetS components is associated with insulin resistance. Based on the facts that levels of RBC, HCT and $\mathrm{Hb}$ are significantly associated with insulin resistance, ${ }^{10} 1224$ we hypothesised that increased levels of erythrocyte parameters tested in this study may be indicative of the development of insulin resistance.

Several studies have demonstrated an association between RBC levels and MetS, indicating that the RBC variable is a potential haematological marker for early detection of MetS. ${ }^{14-16}$ Our results revealed that the highest tertiles of RBC were associated with MetS in women, consistent with a recent study. ${ }^{25}$ The pathogenesis of insulin resistance may, in part, be causative of the association between RBC levels and MetS. Aoki et al reported that insulin can stimulate the proliferation and differentiation of erythropoietic cells by binding receptors on the cell surface. ${ }^{26}$ It was suggested that insulin and insulin growth factors I and II can promote the proliferation and differentiation of erythroid progenitors in human bone marrow and the circulation. ${ }^{27-29}$ Alternatively, the relationship between RBC levels and MetS may be a result of iron overload. It was reported that iron overload was associated with insulin resistance, ${ }^{30}$ and excessive body iron storage interfered with insulin mediated effects, while bloodletting improved insulin sensitivity. ${ }^{31}$ Bozzini et al found that iron overload was strongly associated with obesity and dyslipidaemia, and serum ferritin tests would help identify a subgroup of individuals at risk for insulin resistance associated with hepatic iron overload. $^{32}$ Additionally, erythrocyte fatty acids may be another linking factor between RBC levels and MetS. Novgorodtseva et al found that the development of MetS was accompanied by changes to the composition of erythrocyte fatty acids. ${ }^{33}$ Zong et al also demonstrated that erythrocyte fatty acids in the de novo lipogenesis pathway were independently associated with an elevated risk of MetS. ${ }^{34}$ Fatty acid composition in erythrocytes may affect insulin sensitivity in individuals with MetS. This may be the underlying mechanism linking insulin resistance to changes in fatty acid composition of RBCs in individuals with MetS. ${ }^{35}$

$\mathrm{Hb}$, another important erythrocyte parameter, has been reported to be associated with MetS in both cross sectional and cohort studies. ${ }^{14161736}$ An 8year follow-up cohort study conducted in Japan detected that the highest and third quartiles of $\mathrm{Hb}$ concentration were associated with an increased risk of MetS incidence compared with the lowest quartiles of $\mathrm{Hb}$ concentration in men, but there was no association observed in women. ${ }^{17}$ In general, our findings are consistent with those of previous reports. In our study, the ORs for MetS increased across the 
Table 3 OR of erythrocyte parameters associated with metabolic syndrome stratified by sex

\begin{tabular}{|c|c|c|c|c|}
\hline \multirow[b]{2}{*}{ Variable } & \multicolumn{2}{|l|}{ Men } & \multicolumn{2}{|l|}{ Women } \\
\hline & OR $(95 \% \mathrm{Cl})$ & $P$ value & OR $(95 \% \mathrm{Cl})$ & $P$ value \\
\hline Age & 0.999 (0.986 to 1.013$)$ & 0.930 & 1.012 (0.998 to 1.025$)$ & 0.088 \\
\hline WC & 1.157 (1.121 to 1.194$)$ & $<0.001$ & 1.130 (1.103 to 1.158$)$ & $<0.001$ \\
\hline SBP & 1.025 (1.012 to 1.039$)$ & $<0.001$ & 1.041 (1.031 to 1.051$)$ & $<0.001$ \\
\hline DBP & 1.025 (1.006 to 1.045$)$ & 0.011 & 1.030 (1.013 to 1.047$)$ & 0.001 \\
\hline TG & 3.240 (2.697 to 3.893 ) & $<0.001$ & 1.518 (1.269 to 1.817$)$ & $<0.001$ \\
\hline HDL-C & $0.026(0.012$ to 0.054$)$ & $<0.001$ & 0.001 (0.001 to 0.003$)$ & $<0.001$ \\
\hline FPG & 1.725 (1.410 to 2.111$)$ & $<0.001$ & 2.221 (1.836 to 2.687 ) & $<0.001$ \\
\hline ALT & 0.996 (0.980 to 1.012$)$ & 0.620 & $1.002(0.990$ to 1.014$)$ & 0.757 \\
\hline AST & 1.016 (0.988 to 1.045$)$ & 0.260 & & \\
\hline GGT & 1.001 (0.997 to 1.006$)$ & 0.492 & 1.005 (1.000 to 1.010$)$ & 0.064 \\
\hline BMI & 0.976 (0.907 to 1.049$)$ & 0.506 & 1.015 (0.958 to 1.076$)$ & 0.620 \\
\hline $\mathrm{TC}$ & & & 1.243 (1.061 to 1.455$)$ & 0.007 \\
\hline LDL-C & & & 0.992 (0.903 to 1.090$)$ & 0.867 \\
\hline UA & 1.001 (0.999 to 1.003$)$ & 0.291 & 1.001 (0.999 to 1.002$)$ & 0.303 \\
\hline WBC & 1.044 (0.935 to 1.165$)$ & 0.447 & 1.063 (0.959 to 1.178$)$ & 0.248 \\
\hline PLT & 1.002 (0.999 to 1.005$)$ & 0.202 & 1.001 (0.998 to 1.003$)$ & 0.639 \\
\hline HbAlc & 0.856 (0.649 to 1.130$)$ & 0.273 & $0.747(0.572$ to 0.976$)$ & 0.032 \\
\hline \multicolumn{5}{|l|}{$\mathrm{RBC}$} \\
\hline Q1 & Reference & & Reference & \\
\hline Q2 & 0.940 (0.613 to 1.443$)$ & 0.779 & $1.070(0.743$ to 1.541$)$ & 0.716 \\
\hline Q3 & 1.207 (0.771 to 1.889$)$ & 0.410 & 1.718 (1.173 to 2.515$)$ & 0.005 \\
\hline \multicolumn{5}{|l|}{ HCT } \\
\hline Q1 & Reference & & Reference & \\
\hline Q2 & 0.771 (0.482 to 1.234$)$ & 0.279 & 1.419 (0.933 to 2.159$)$ & 0.102 \\
\hline Q3 & 0.968 (0.606 to 1.547$)$ & 0.893 & 1.407 (0.896 to 2.208$)$ & 0.138 \\
\hline \multicolumn{5}{|l|}{$\mathrm{Hb}$} \\
\hline Q1 & Reference & & Reference & \\
\hline Q2 & $1.921(1.170$ to 3.151$)$ & 0.010 & 1.538 (1.008 to 2.348$)$ & 0.046 \\
\hline Q3 & 1.992 (1.198 to 3.312$)$ & 0.008 & 1.665 (1.075 to 2.578$)$ & 0.022 \\
\hline \multicolumn{5}{|l|}{ RDW } \\
\hline Q1 & Reference & & Reference & \\
\hline Q2 & 1.114 (0.757 to 1.639$)$ & 0.583 & 0.787 (0.571 to 1.085$)$ & 0.144 \\
\hline Q3 & 2.725 (1.915 to 3.878$)$ & $<0.001$ & 1.057 (0.753 to 1.484$)$ & 0.750 \\
\hline
\end{tabular}

The bolded values represented $P$ value $<0.05$.

Statistical analysis by binary logistic regression with adjustments for potential confounders (the statistically significant variables in table 1). Men: adjusted for age, WC, SBP, DBP, TG, HDL-C, FPG, ALT, AST, GGT, BMI, UA, WBC, PLT and HbAlc. Women: adjusted for age, WC, SBP, DBP, TG, HDL-C, FPG, ALT, GGT, BMI, TC, LDL-C, UA, WBC, PLT and HbAlc.

ALT, alanine transaminase; AST, aspartate aminotransferase; BMI, body mass index; DBP, diastolic blood pressure; FPG, fasting plasma glucose; GGT, $\gamma$-glutamyl transferase; Hb, haemoglobin; HbAlc, glycated haemoglobin A1c; HCT, haematocrit; HDL-C, high density lipoprotein cholesterol; LDL-C, low density lipoprotein cholesterol; PLT, platelets; RBC, red blood cell; RDW, red blood cell distribution width; SBP, systolic blood pressure; TC, total cholesterol; TG, triglyceride; UA, uric acid; WBC, white blood cell; WC, waist circumference.

successive tertiles of $\mathrm{Hb}$ among men; however, no similar trend was observed in women. The following mechanisms may be regarded as the causes of the association between $\mathrm{Hb}$ and MetS: $\mathrm{Hb}$ is a well known carrier and buffer of nitric oxide (NO), and can regulate the endothelial function of blood vessels by modulating NO levels in blood. ${ }^{37}$
Furthermore, $\mathrm{Hb}$ and various compounds of $\mathrm{NO}$ modulate the affinity between $\mathrm{Hb}$ and oxygen in blood, which can lead to vascular endothelial dysfunction. ${ }^{38}$ It has been found that vascular endothelial dysfunction was associated with MetS. ${ }^{39} 40$ In addition, Hb plays a key role in regulating sCD40L levels, ${ }^{41}$ and $\mathrm{SCD} 40 \mathrm{~L}$ has been shown 
to participate in thrombus formation and inflammation, which is an independent risk factor for atherosclerosis and MetS. ${ }^{42}$ Another possibility linking $\mathrm{Hb}$ and MetS may be adiponectin. Previous studies showed that higher Hb levels were closely related to lower adiponectin levels, ${ }^{43} 44$ and lower levels of adiponectin significantly increased the risk for MetS, respectively. Finally, insulin resistance may also be involved in the association between $\mathrm{Hb}$ and MetS. ${ }^{812}$

RDW, a common index of routine blood examinations, represents a measure of heterogeneity in the size of circulating erythrocytes. A high RDW index indicates greater heterogeneity in the size of circulating erythrocytes in a subject. In this study, men in the highest tertiles of RDW (>13.2\%) had a 2.75-fold increased risk for MetS. Multiple groups previously showed that elevated RDW was associated with MetS. ${ }^{45}{ }^{46}$ For instance, Laufer et al demonstrated that RDW $\geq 14 \%$ was independently associated with an increased risk for MetS development ${ }^{45}$; Sanchez-Chaparro et al reported that the highest quartile of RDW (>14\%) was linked with MetS after adjusting for potential confounders. ${ }^{46}$ Moreover, a recent study showed that RDW is a potential metabolic marker for the detection of metabolic diseases. ${ }^{47}$ To date, the mechanism for the association between RDW and MetS remains unknown; however, chronic inflammation linked to RDW may play an important role. MetS has previously been associated with chronic inflammation, ${ }^{9}$ and RDW reflects an underlying inflammatory state. ${ }^{13}$ Pierce et al have proved that proinflammatory cytokines can inhibit erythropoietin induced erythrocyte maturation, which may lead to an elevation in RDW. ${ }^{48}$

Our study was conducted in the Pearl River Delta region of China, and it may imply that the generalisability of our results is limited to this region. Additionally, participants with a history of cardiovascular diseases, severe liver or kidney dysfunction, tumours or severe inflammatory diseases were excluded, so our results are not applicable to these subjects.

There were several limitations in this study. First, the present study was designed as a cross sectional study; therefore, direct causation cannot be concluded from the results. Supplementary information about the lifestyle of the subjects was not collected and hence factors such as smoking, physical exercise and dietary could not be included in the adjustments of our multivariate logistic regression analyses.

\section{CONCLUSIONS}

In our study, MetS was more prevalent in women than in males. The association between erythrocyte parameters and MetS differed between the sexes: $\mathrm{RBC}$ and $\mathrm{Hb}$ were identified as risk factors for MetS in women and $\mathrm{Hb}$ and RDW as risk factors in men. This has important clinical implications for health professionals. Erythrocyte parameters may serve as effective indices for the early detection of the risk and treatment of MetS on a sex dependent basis.
Acknowledgements We gratefully acknowledge the staff of local Community Health Service Agencies, for their kind assistance in data collection.

Contributors LLH and PXW conducted the data analyses. LLH, NL, XXW, LYF and $X W$ drafted the manuscript. PXW and DMD finalised the manuscript with inputs from all authors. All authors contributed to the development of the study framework, interpretation of the results, revisions of successive drafts of the manuscript and approved the version submitted for publication.

Funding This study was supported by the Guangzhou 121 Talents Programme (GZRSH-2014-2048), the Science and Technology Program of Guangzhou (201607010136, 201510010109) and the National Science Foundation of China (81402716)

\section{Competing interests None declared.}

Patient consent Obtained.

Ethics approval The study was approved by the ethics committee of Guangdong Sociological Society.

Provenance and peer review Not commissioned; externally peer reviewed.

Data sharing statement This database was first used in this study and belongs to our team. Permission should be sought from all authors to share any data.

Open Access This is an Open Access article distributed in accordance with the Creative Commons Attribution Non Commercial (CC BY-NC 4.0) license, which permits others to distribute, remix, adapt, build upon this work non-commercially, and license their derivative works on different terms, provided the original work is properly cited and the use is non-commercial. See: http://creativecommons.org/ licenses/by-nc/4.0/

(c) Article author(s) (or their employer(s) unless otherwise stated in the text of the article) 2018. All rights reserved. No commercial use is permitted unless otherwise expressly granted.

\section{REFERENCES}

1. Grundy SM, Cleeman JI, Daniels SR, et al. Diagnosis and management of the metabolic syndrome: an American Heart Association/National Heart, Lung, and Blood Institute scientific statement. Circulation 2005;112:2735-52.

2. Alberti KG, Eckel RH, Grundy SM, et al. Harmonizing the metabolic syndrome: a joint interim statement of the International Diabetes Federation Task Force on Epidemiology and Prevention; National Heart, Lung, and Blood Institute; American Heart Association; World Heart Federation; International Atherosclerosis Society; and International Association for the Study of Obesity. Circulation 2009;120:1640-5.

3. Mottillo S, Filion KB, Genest J, et al. The metabolic syndrome and cardiovascular risk a systematic review and meta-analysis. J Am Coll Cardiol 2010;56:1113-32.

4. Gami AS, Witt BJ, Howard DE, et al. Metabolic syndrome and risk of incident cardiovascular events and death: a systematic review and meta-analysis of longitudinal studies. J Am Coll Cardiol 2007;49:403-14.

5. Miller JM, Kaylor MB, Johannsson M, et al. Prevalence of metabolic syndrome and individual criterion in US adolescents: 2001-2010 National Health and Nutrition Examination Survey. Metab Syndr Relat Disord 2014;12:527-32.

6. Peer N, Lombard C, Steyn K, et al. High prevalence of metabolic syndrome in the Black population of Cape Town: The Cardiovascular risk in black South Africans (CRIBSA) study. Eur J Prev Cardiol 2015;22:1036-42.

7. Lovre D, Mauvais-Jarvis F. Trends in prevalence of the metabolic syndrome. JAMA 2015;314:950-1.

8. González AS, Guerrero DB, Soto MB, et al. Metabolic syndrome, insulin resistance and the inflammation markers $\mathrm{C}$-reactive protein and ferritin. Eur J Clin Nutr 2006;60:802-9.

9. Musani SK, Vasan RS, Bidulescu A, et al. Aldosterone, C-reactive protein, and plasma B-type natriuretic peptide are associated with the development of metabolic syndrome and longitudinal changes in metabolic syndrome components: findings from the Jackson Heart Study. Diabetes Care 2013;36:3084-92.

10. Ellinger VC, Carlini LT, Moreira RO, et al. Relation between insulin resistance and hematological parameters in a Brazilian sample. Arq Bras Endocrinol Metabol 2006;50:114-7.

11. Mardi T, Toker S, Melamed S, et al. Increased erythropoiesis and subclinical inflammation as part of the metabolic syndrome. Diabetes Res Clin Pract 2005;69:249-55. 
12. Tabara $\mathrm{Y}$, Igase $\mathrm{M}$, Saito I, et al. Association of hematological parameters with insulin resistance, insulin sensitivity, and asymptomaticcerebrovascular damage: The J-SHIP Toon Health Study. Clin Hemorheol Microcirc 2013;55:297-311.

13. Lippi G, Targher G, Montagnana M, et al. Relation between red blood cell distribution width and inflammatory biomarkers in a large cohort of unselected outpatients. Arch Pathol Lab Med 2009;133:628-32.

14. Kawamoto R, Tabara Y, Kohara K, et al. Hematological parameters are associated with metabolic syndrome in Japanese communitydwelling persons. Endocrine 2013;43:334-41.

15. Wu S, Lin H, Zhang C, et al. Association between erythrocyte parameters and metabolic syndrome in urban Han Chinese: a longitudinal cohort study. BMC Public Health 2013;13:1-8.

16. Nebeck K, Gelaye B, Lemma S, et al. Hematological parameters and metabolic syndrome: findings from an occupational cohort in Ethiopia. Diabetes Metab Syndr 2012;6:22-7.

17. Hashimoto $Y$, Tanaka M, Kimura T, et al. Hemoglobin concentration and incident metabolic syndrome: a population-based large-scale cohort study. Endocrine 2015;50:390-6.

18. Vayá A, Carmona P, Badia N, et al. Association between high red blood cell distribution width and metabolic syndrome. Influence of abdominal obesity. Clin Hemorheol Microcirc 2011;47:75-7.

19. Sidorenkov O, Nilssen O, Grijbovski AM. Metabolic syndrome in Russian adults: associated factors and mortality from cardiovascular diseases and all causes. BMC Public Health 2010;10:1-10.

20. Kozan O, Abaci A, Abaci A, et al. Prevalence of the metabolic syndrome among Turkish adults. Eur J Clin Nutr 2007:61:548-53.

21. Tao LX, Li X, Zhu HP, et al. Association of hematological parameters with metabolic syndrome in Beijing adult population: a longitudinal study. Endocrine 2014;46:485-95.

22. Yang $X$, Tao Q, Sun F, et al. The impact of socioeconomic status on the incidence of metabolic syndrome in a Taiwanese health screening population. Int J Public Health 2012;57:551-9.

23. Wang YY, Lin SY, Liu PH, et al. Association between hematological parameters and metabolic syndrome components in a Chinese population. J Diabetes Complications 2004;18:322-7.

24. Choi KM, Lee J, Kim YH, et al. Relation between insulin resistance and hematological parameters in elderly Koreans-Southwest Seoul (SWS) Study. Diabetes Res Clin Pract 2003;60:205-12.

25. Wang $\mathrm{T}$, Wang $\mathrm{H}$. Study of the relationship between female metabollic syndrome and its related blood indexes in Guangzhou. Medical Innovation of China 2016;13:61-4.

26. Aoki I, Taniyama M, Toyama K, et al. Stimulatory effect of human insulin on erythroid progenitors (CFU-E and BFU-E) in human CD34+ separated bone marrow cells and the relationship between insulin and erythropoietin. Stem Cells 1994;12:329-38.

27. Bersch N, Groopman JE, Golde DW. Natural and biosynthetic insulin stimulates the growth of human erythroid progenitors in vitro. $\mathrm{J}$ Clin Endocrinol Metab 1982;55:1209-11.

28. Miyagawa S, Kobayashi M, Konishi N, et al. Insulin and insulin-like growth factor I support the proliferation of erythroid progenitor cells in bone marrow through the sharing of receptors. $\mathrm{Br} J$ Haematol 2000;109:555-62.

29. Dainiak N, Kreczko S. Interactions of insulin, insulinlike growth factor $\mathrm{II}$, and platelet-derived growth factor in erythropoietic culture. J Clin Invest 1985;76:1237-42.
30. Martínez-García MA, Luque-Ramírez M, San-Millán JL, et al. Body iron stores and glucose intolerance in premenopausal women: role of hyperandrogenism, insulin resistance, and genomic variants related to inflammation, oxidative stress, and iron metabolism. Diabetes Care 2009;32:1525-30.

31. Fernández-Real JM, Peñarroja G, Castro A, et al. Blood letting in high-ferritin type 2 diabetes: effects on insulin sensitivity and betacell function. Diabetes 2002;51:1000-4.

32. Bozzini C, Girelli D, Olivieri O, et al. Prevalence of body iron excess in the metabolic syndrome. Diabetes Care 2005;28:2061-3.

33. Novgorodtseva TP, Karaman YK, Zhukova NV, et al. Composition of fatty acids in plasma and erythrocytes and eicosanoids level in patients with metabolic syndrome. Lipids Health Dis 2011;10:82.

34. Zong G, Zhu J, Sun L, et al. Associations of erythrocyte fatty acids in the de novo lipogenesis pathway with risk of metabolic syndrome in a cohort study of middle-aged and older Chinese. Am J Clin Nutr 2013;98:319-26.

35. Djoussé L, Matthan NR, Lichtenstein AH, et al. Red blood cell membrane concentration of cis-palmitoleic and cis-vaccenic acids and risk of coronary heart disease. Am J Cardiol 2012;110:539-44.

36. Lohsoonthorn V, Jiamjarasrungsi W, Williams MA. Association of hematological parameters with clustered components of metabolic syndrome among professional and office workers in Bangkok, Thailand. Diabetes Metab Syndr 2007;1:143-9.

37. Schiffrin EL. Oxidative stress, nitric oxide synthase, and superoxide dismutase: a matter of imbalance underlies endothelial dysfunction in the human coronary circulation. Hypertension 2008;51:31-2.

38. Zinchuk VV, Pronko TP, Lis MA. Blood oxygen transport and endothelial dysfunction in patients with arterial hypertension. Clin Physiol Funct Imaging 2004;24:205-11.

39. Wei Y, Liu G, Yang J, et al. The association between metabolic syndrome and vascular endothelial dysfunction in adolescents. Exp Ther Med 2013;5:1663-6.

40. Tsuji S, Node K. [Vascular endothelial dysfunction as a mechanistic factor for metabolic syndrome]. Nihon Rinsho 2011;69 Suppl 1:295

41. Kutlu M, Sonmez A, Genc H, et al. Relationship between hemoglobin and CD40 ligand in prediabetes. Clin Invest Med 2009;32:244-50.

42. Missiou A, Wolf $D$, Platzer I, et al. CD40L induces inflammation and adipogenesis in adipose cells a potential link between metabolic and cardiovascular disease. Thromb Haemost 2010;103:788-96.

43. Kawamoto R, Tabara $\mathrm{Y}$, Kohara K, et al. Hemoglobin is associated with serum high molecular weight adiponectin in Japanese community-dwelling persons. J Atheroscler Thromb 2011;18:182-9.

44. Ben Ali S, Jemaa R, Ftouhi B, et al. Adiponectin and metabolic syndrome in a Tunisian population. Inflammation 2012;35:828-33.

45. Laufer Perl M, Havakuk O, Finkelstein A, et al. High red blood cell distribution width is associated with the metabolic syndrome. Clin Hemorheol Microcirc 2015;63:1-9.

46. Sanchez-Chaparro MA, Calvo-Bonacho E, Gonzalez-Quintela A, et al. Higher red blood cell distribution width is associated with the metabolic syndrome. Diabetes Care 2010;33:e40.

47. Perna S, Peroni G, Monteferrario F, et al. PP249-SUN: The role of red blood cell distribution width in metabolic syndrome. A crosssectional study in elderly. Clin Nutr 2014;33:S112.

48. Pierce CN, Larson DF. Inflammatory cytokine inhibition of erythropoiesis in patients implanted with a mechanical circulatory assist device. Perfusion 2005;20:83-90. 